\title{
A usability study of a multicomponent video game-based training for older adults
}

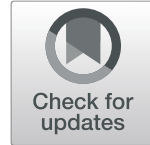

\author{
Manuela Adcock ${ }^{1 *} \mathbb{D}$, Floriana Sonder ${ }^{1}$, Alexandra Schättin ${ }^{1}$, Federico Gennaro ${ }^{1}$ and Eling D. de Bruin ${ }^{1,2}$
}

\begin{abstract}
Background: Aging is often accompanied by a decline in sensory, motor and cognitive functions. These age- and lifestyle-related impairments may lead to reduced daily life functioning including gait disturbances, falling and injuries. Most daily life activities, e.g. walking, are tasks which require the concurrent interplay of physical and cognitive functions. Promising options for combined physical-cognitive training are video game-based physical exercises, so-called exergames. This study aimed to [i] determine the usability of a newly developed multicomponent exergame and [ii] explore its effects on physical functions, cognition and cortical activity.

Methods: Twenty-one healthy and independently living older adults were included (10 female, $71.4 \pm 5.8$ years, range: 65-91) and performed 21 training sessions (each $40 \mathrm{~min}$ ) over seven weeks. The multicomponent exergame included strength and balance training with Tai Chi-inspired and dance exercises. Participants rated the usability of the exergame (System Usability Scale) and reported on their emotional experience (Game Experience Questionnaire). Attendance and attrition rates were calculated to determine training compliance. Before and after the intervention, physical and cognitive functions as well as resting state electroencephalography (EEG) were assessed.
\end{abstract}

Results: Results showed a high training attendance rate $(87.1 \%, 18 / 21$ training sessions on average) and a low attrition rate $(9.5 \%, 2$ drop-outs). System usability was rated high with a mean score of $75 / 100$. Affective game experience was rated favorable. Gait speed under dual-task condition, lower extremity muscle strength and reaction times in a cognitive task (divided attention) showed significant improvements $(p<.05)$. No significant pre-post differences were found for resting state EEG.

Conclusions: The newly developed exergame seems usable for healthy older adults. Nevertheless, some aspects of the exergame prototype can and should be improved. The training showed to positively influence physical and cognitive functions in a small convenience sample. Future trials are warranted which evaluate the feasibility and usability of the exergame training in a more "real-life" in-home setting and assess the behavioral and neuroplastic changes in a larger population after a longer training period with comparison to a control group.

Keywords: Physical-cognitive intervention, Exergame, Healthy aging, Fall prevention, Elderly, Older adults, Usability, Game experience

\section{Background}

Age- and lifestyle-associated degenerative changes cause reduced daily life functioning including gait impairments and a higher fall risk in elderly [1]. Falling can lead to injuries, movement restrictions, loss of independence, social isolation, depression and a general decrease in wellbeing and quality of life [2]. Current numbers

\footnotetext{
* Correspondence: momlin@hest.ethz.ch

${ }^{1}$ Department of Health Sciences and Technology, Institute of Human

Movement Sciences and Sport, ETH Zurich, Leopold-Ruzicka-Weg 4, 8093

Zurich, Switzerland

Full list of author information is available at the end of the article
}

demonstrate that one out of three people aged 65 years and older fall annually and $20-30 \%$ of falls result in injury and hospitalization [3, 4]. Considering the significant impact on the individual lives of the growing elderly population as well as on healthcare costs, a strong need exists to examine interventions that aim to support healthy aging and prevent falls. For successful falls prevention, causes and risk factors of falling must be targeted. It is well known that age-related declines in sensorimotor systems lead to muscle weakness, reduced muscle strength and increased balance problems and,

(c) The Author(s). 2020 Open Access This article is distributed under the terms of the Creative Commons Attribution 4.0 International License (http://creativecommons.org/licenses/by/4.0/), which permits unrestricted use, distribution, and reproduction in any medium, provided you give appropriate credit to the original author(s) and the source, provide a link to the Creative Commons license, and indicate if changes were made. The Creative Commons Public Domain Dedication waiver (http://creativecommons.org/publicdomain/zero/1.0/) applies to the data made available in this article, unless otherwise stated. 
therefore, to gait disturbances as well as a higher risk of falling $[5,6]$. Exercise interventions, which aim to improve physical functions such as strength or balance training, showed to reduce fall rates and risks [7-9].

Age-associated changes occur also on neuronal level. The aging brain exhibits structural changes in gray and white matter [10-12] and functional changes in cortical oscillatory activity patterns [13]. An age-related "slowing" in brain activity is described (with an increase in slow frequency ranges and a decrease in higher frequencies e.g. alpha frequency) [13, 14]. On a cellular level, physiological aging is characterized by a loss of synaptic contacts and an apoptosis of neurons which can lead to a decline of sensorimotor and cognitive functions [13]. Most human daily life activities, including walking, require physical and cognitive resources. Safe and stable walking, especially in a demanding environment, is based on intact continuous interactions of physical and cognitive functions [15-19]. With age-related changes affecting the whole system from brain to muscles, the performance of physical-cognitive activities is impaired which, in turn, is considered a main risk factor for falling in older adults [20-22]. Therefore, a combined physicalcognitive training is important for effective fall prevention [23, 24]. Regular physical activity in older age effects gait stability, health status and general well-being [2527]. However, in most existing training approaches for fall prevention, no explicit attention is paid to cognitive functions and the physical-cognitive interplay. A promising option for simultaneous training of physical and cognitive functions are interactive video game-based physical exercises or so called exergames [28].

Exergames are defined as "any types of video games that require the player to be physically active and move to play the game" [29]. Due to a combination of physical exercises with cognitive stimulation, exergame training might be closer to daily life requirements. Another advantage of exergames may relate to the "gamification" approach of training. Several studies demonstrated that exergames have a high motivational potential by providing enjoyable gameplay [30, 31]. Moreover, exergame training can be applied in diverse settings (e.g. home-based training). Purpose developed exergames for public health and disease prevention are well advised to be designed according to target users' expectations and needs [29]. Following a user-centered design approach, acceptability and training adherence seem to increase and this, in turn, can enhance specific training effects $[32,33]$.

Considering older adults' needs and requirements, the Active@Home project, an international project of the Ambient Assisted Living Association, developed an exergame prototype by incorporating theoretical background from human movement sciences and neuropsychology and the art of game design. The Active@Home exergame enables multicomponent training including strength, balance and cognitive training components and focuses on supporting healthy aging including fall prevention. Before conducting full-scale studies, the usability of this newly developed exergame as well as its acceptance by older adults should be tested [34]. Especially for training interventions, a usable solution for the target population is mandatory to ensure training compliance and benefits. Therefore, the primary aim of this study was to determine the usability of the newly developed Active@Home exergame. The secondary aim was to explore whether the exergame intervention can influence physical and/or cognitive functions as well as cortical activity at rest. We hypothesized a high usability of the newly developed exergame training for older adults due to following a user-centered development and design approach and based on findings from literature showing a high usability of virtual reality and technology-based training for the older population [32, 35]. Moreover, positive effects on gait parameters, balance, functional muscle strength as well as attentional and executive functions and brain activity in alpha frequency have been hypothesized.

\section{Methods}

\section{Study design and participants}

Campbell and colleagues recommend an iterative phased approach starting with exploratory trials (phase II studies) before conducting definitive randomized controlled trials (phase III studies) [34]. This phase II exploratory study used a single arm pre-post testing design. From March to May 2017, potential participants were recruited through public advertisements in local newspapers and from the pensioner community ETH Zurich (PVETH, Switzerland). Assessments and intervention were performed at ETH Hönggerberg (Zurich, Switzerland). Measurements were conducted before (June 2017) and after (September 2017) the intervention period. In addition, a between-measurement consisting of two questionnaires was performed after the first week of training. Before the intervention period started, participants wore an activity monitoring device (StepWatch) for 1 week. The ETH Zurich Ethics Committee (Zurich, Switzerland) granted ethical approval (protocol number EK 2017-N-06). All participants were fully informed prior to participation and signed an informed consent form according to the Declaration of Helsinki before conducting any measurement.

The potential participants were screened using the Montreal Cognitive Assessment (MOCA) to assess cognitive status. Furthermore, the participants completed a health questionnaire including anthropometric data and questions about their health, medical history and physical activity level. Participants fulfilling all of the 
following inclusion criteria were eligible for the study: (1) age $\geq 65$ years, (2) living independently, (3) healthy (self-reported), (4) able to walk at least $20 \mathrm{~m}$ with or without walking aids. Participants exhibiting at least one of the following criteria were excluded from the study: (1) mobility impairments that prevent from training participation, (2) severe and uncontrolled health problems (e.g. recent cardiac infarction, uncontrolled diabetes or hypertension), (3) orthopaedic disease that prevents from training participation, (4) neurological disease (e.g. history of stroke or epilepsy, Parkinson's disease), (5) Alzheimer disease or other forms of dementia, (6) acute severe, rapidly progressive or terminal illness, (7) cognitive impairments (MOCA < 26 points), (8) intake of any psychoactive substances (e.g. neuroleptics, antidepressants), (9) high alcohol, caffeine or nicotine consumption. The minimal intended study sample size of 20 participants was based on previously conducted feasibility and usability studies [35, 36] and on a practitioner's guide [37].

\section{Exergame intervention}

From June to September 2017, the participants performed three training sessions per week for 7 weeks resulting in a maximum of 21 training sessions. The training sessions were scheduled individually from Monday to Friday with a guideline of no more than one training session per day. The 21 training sessions were distributed within a period of seven to 9 weeks as a maximum of 2 weeks holiday interruption was allowed in between. Each session consisted of $40 \mathrm{~min}$ training with the newly developed Active@Home exergame prototype including Tai Chi-inspired training (20-30 $\mathrm{min})$ and dance exercises $(10 \mathrm{~min})$. Tai Chi-inspired exercises were a combination of lower-limb and core strength exercises and Tai Chi elements in three different stance positions (squat, plié, and lunge). Tai Chi-like movements were used as this ancient Chinese physical activity is often performed in a semi-squat posture, placing load on the lower limbs and core muscles [38]. These muscles are important for functional movements as walking [39, 40] and are positively influenced by Tai Chi training in the elderly [41]. Beside of increasing muscle strength, Tai chi has been shown to enhance balance and coordinative skills as well as cognitive functions; the later may be due to the cognitively demanding exercises $[38,42,43]$. To ensure optimal training effects, muscle loading recommendations for older adults were applied to the Tai Chiinspired exercises (e.g. time under tension of $6 \mathrm{~s}$ per repetition, a rest of $4 \mathrm{~s}$ between repetitions, 7-9 repetitions per set, a rest of 60 s between sets, a training volume of 2-3 sets per exercise) [44]. Additionally, dance exercises were included in the Active@Home exergame. Dancing exercises were based on common dances as Bachata, Disco Fox, Salsa, Waltz, Cha-Cha-Cha, and Jive and, in general, require motor components of balance, coordination, and agility, but also cognitive resources [45-47]. Dancing and the execution of rapid and welldirected steps has been shown to improve balance, coordinative skills, endurance and cognitive functions [4853]. Both, Tai Chi and dancing are "holistic" and taskoriented physical activities $[54,55]$. The exercises were accentuated with background music [56].

The exergame prototype implemented some basic training principles [57] as a feedback system with a realtime colour code for performance (red colour for bad performance, orange colour for moderate performance, green colour for good performance) and performance scores during and after each exercise. To ensure optimal challenge (optimal load of task demands) and increasing difficulty (progression), several difficulty levels for Tai Chi-inspired and dance exercises were developed. Progression was reached through more complex movements in the Tai Chi-inspired exercises (e.g. additional arm movements, upper body rotations, increased range of motion, longer time in unstable position) and through additional weights (e.g. filled water bottles), while faster and more complex motion sequences were performed in dance exercises.

The game story was about travelling in Europe and to train in several different European cities. To demonstrate the exercises, a virtual instructor was used. The game interface was presented on a TV screen connected to a laptop running the exergame software. For movement evaluation, the participants wore four inertial measurement units (IMUs) providing both accelerometer and gyroscope assessments. The IMUs were connected via Bluetooth to the laptop and attached to participants' wrists and ankles with Velcro straps. Figure 1 shows the training set up. Participants trained alone in the laboratory at ETH Hönggerberg (Zurich, Switzerland) wearing comfortable sports clothes and shoes. Two postgraduate students supported the participants and systematically observed them throughout the intervention. Furthermore, they ensured that the training principles of optimal load and progression were present $[57,58]$. Training intensity was individually adapted to target a moderate to vigorous training level [59, 60]. Intervention characteristics as frequency, duration and training intensity were based on recommendations for fall prevention in elderly [44, 59-61] and on studies showing positive training effects of exergame training in older adults [62].

\section{Primary outcome}

Usability of the newly developed exergame prototype was evaluated using quantitative and qualitative assessments. A mixed method approach was chosen similar to other studies which evaluated the usability of exergames [35]. Questionnaires were completed by participants after three training sessions (between-measurement) and after the intervention period (post-measurement). 


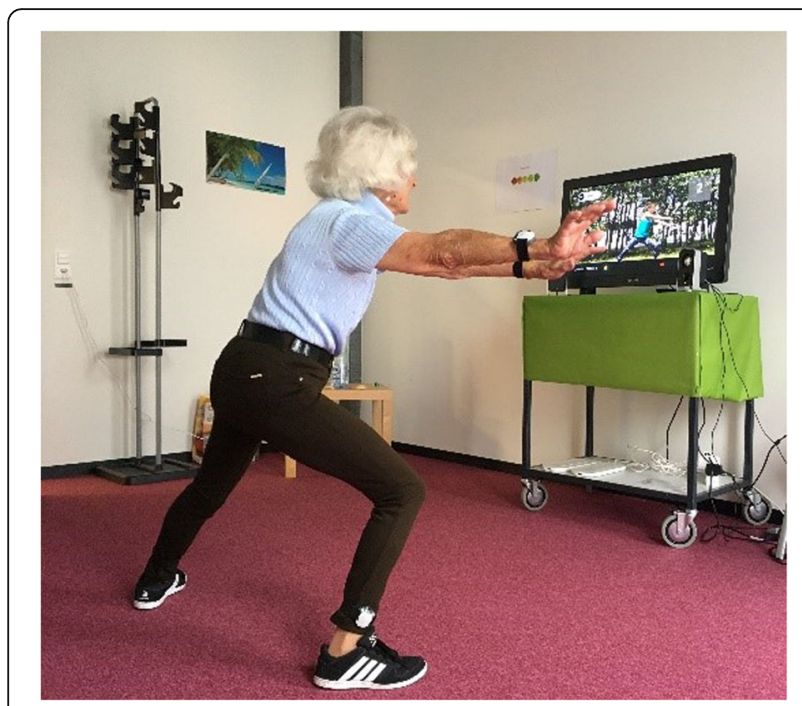

Fig. 1 Set up of the Active@Home exergame training. Participants wore four IMUs at wrists and ankles for movement evaluation. On the TV screen, a virtual instructor presented the training exercise which had to be imitated by the participant. Written consent was obtained from the participant in the picture allowing the usage of the picture for scientific publication

\section{Questionnaires}

The System Usability Scale (SUS) includes 10 items rated on a 5 -point Likert scale $(0=$ "strongly disagree" to $4=$ "strongly agree") and is a validated and reliable scale for evaluating subjective usability of newly developed devices and systems $[63,64]$. The sum of all item scores was multiplied with 2.5 and led to the SUS score ranging between 0 to 100 , whereas higher scores indicate better usability [63]. Based on the verbal categorization rate of Bangor [65], we expected a SUS score $\geq 70$ for an "acceptable system". An additional question was added at the end of the SUS, asking participants about their general opinion of the Active@Home exergame. This question was also rated on a 5-point Likert scale ( $0=$ "I don't like it" to $4=$ "I like it a lot") and the mean was calculated over all participants.

The Game Experience Questionnaire (GEQ) assessed several categories of subjective game experience (competence, immersion, flow, tension, challenge, negative affect, positive affect) $[31,66]$, and includes in total 42 items rated on a 5 -point Likert scale $(0=$ "not at all" to 4 = "extremely"). Competence implies feelings of being successful, strong or skilful in the game. Immersion includes the interest and pleasure of a player in the game. Flow summarizes the feelings of being deeply concentrated and absorbed, forgetting time and losing connection to the world outside the game. Tension includes feelings of annoyance, frustration and pressure. Challenge implies feelings of being stimulated and challenged. Negative affect summarizes feelings related to a bad mood and boredom, whereas positive affect includes feeling of happiness and enjoyment. The GEQ was analyzed by calculating the average rating for each of the seven categories [67]. Two categories involved only negative coded items (tension and negative affect). These two categories were reverse evaluated [66].

\section{Training observation and feedback}

The usability protocol was structured in six categories: (1) functionality and interaction with the system, (2) IMUs, (3) design, (4) training principles, (5) exercises, and (6) emotions. It was filled in by the supervisors observing the participants during their training sessions. The participants were requested to "think aloud" and mention all thoughts that came to their mind while using the exergame [68]. Furthermore, the protocol included general feedback from participants. The collected observations and statements were separated in positive and negative aspects for each category.

\section{Training compliance}

An attendance protocol, filled in by the supervisors after each training session, was used to record the number of visited training sessions. The adherence rate was calculated using the number of visited training sessions as percentage of the maximum possible training sessions [36, 69]. A $70 \%$ attendance rate (15 visited out of 21 total training sessions planned) was considered "being adherent" to the training program $[69,70]$. For attrition, the number of participants lost during the trial was recorded (drop-outs) and calculated as a percentage of the total sample size. Considering the median rate for attrition in fall prevention interventions for clinical trials, a 10\% attrition rate (two drop-outs) was regarded acceptable [70]. Drop-outs were not considered in the calculation of the adherence rate. Reasons for non-adherence and drop-outs were, when given by the participants, recorded with a special interest in mal-compliance related to usability issues.

\section{Secondary outcomes}

As secondary outcomes, physical and cognitive functions as well as cortical activity were measured before and after the intervention period (pre-measurement and post-measurement, respectively).

\section{Physical functions}

Parameters of gait kinematics were assessed using the Physilog5 IMU (Gait Up Sàrl, Lausanne, Switzerland), which has been shown to reliably measure gait performance [71]. The Physilog5 IMUs were fixed to the top of the right and left forefoot of participants using elastic straps. A USB port allows data transfer to the computer for further data analysis. A walking protocol involving at least 50 gait cycles was used [72]. Participants walked a 
straight distance of $80 \mathrm{~m}$ under two conditions: (1) single-task condition (ST): participants were instructed to walk at preferred speed without talking; (2) dual-task condition (DT): participants had to walk at preferred speed and simultaneously count backwards (cognitive task) in steps of seven from a randomly given number between 200 and 250. In this condition, participants were asked to perform both tasks concurrently and not to prioritise one task at the cost of the other. This is a common method to measure multitasking capabilities $[73,74]$. Two walking steps for initiation and termination were discarded in order to analyse steady state walking [75]. Speed $[\mathrm{m} / \mathrm{s}]$, cadence [steps $/ \mathrm{min}]$, stride length $[\mathrm{m}]$, and minimal toe clearance $[\mathrm{cm}]$ were evaluated and expressed as mean values of both legs in the two walking conditions. For each parameter, the dual-task cost (DTC) of walking was calculated as a percentage of loss of the $\mathrm{DT}$ relative to the ST condition according to the formula: DTC $[\%]=(\mathrm{ST}-\mathrm{DT}) / \mathrm{ST} \times 100[76]$.

To assess lower extremity functioning, the Short Physical Performance Battery (SPPB) was applied [77, 78]. A maximum of 12 points can be achieved where a low score is associated with a higher risk of falls [79]. The SPPB includes a balance test, a $4 \mathrm{~m}$-walk test and a 5chair rises test (maximally 4 points for each subtests). Details about the protocol can be found elsewhere [78]. In line with Eggenberger et al. [73], we extended the balance test with two additional tasks to avoid ceiling effects. The first additional task was a 20s single-leg stance (with preferred leg) where two points were achieved for reaching 20s, one point for $10-20$ s and zero points for $<$ 10s. The second additional task was a single-leg stance with eyes closed (with preferred leg) where one point was assigned for every $5 \mathrm{~s}$ of successful task achievement. For the extended version of the SPPB, the maximum point score is unlimited. For the analysis, the total score of the extended SPPB was calculated as well as the score for each subtest (balance score, $4 \mathrm{~m}$-gait score, 5-chair rises score). For pre- and post-measurement comparison, time measures of the $4 \mathrm{~m}$-gait test and the 5 -chair rises test were also evaluated.

\section{Cognitive functions}

Higher cognitive functions such as working memory, divided and selective attention, inhibition and mental flexibility were assessed using four tests of the computerized test battery Test of Attentional Performance (TAP, DTAP 2.3 VL, PSYTEST, Psychologische Testsysteme, Herzogenrath, Germany). The TAP is a valid assessment of different attentional and executive functions [80]. The following tests were performed on a computer using two answer buttons: Working memory (difficulty level 3), Divided attention, GoNogo (1 out of 2), Set-shifting (alternating letters and numbers). Each test was preceded by a short familiarization session. Details about this protocol can be found elsewhere [80]. For each of the four tests, reaction times $[\mathrm{ms}]$, number of errors and omissions were analysed.

\section{Cortical activity and analysis}

In order to assess cortical oscillatory activity, 5 minutes of resting state electroencephalography (EEG) were recorded at $500 \mathrm{~Hz}$ sampling rate, using a 20-channels dryelectrodes cap (ENOBIO 20, Neuroelectrics, Barcelona, Spain) placed according to the international 10-20 system [81] and referenced using the Driven-Right-Leg (DRL) / Common Mode Sense (CMS) technique (two external electrodes placed on either side of the left earlobe with an ear-clip). Before electrode placement on the forehead and earlobe, the skin was prepared with abrasive paste $(\mathrm{H}+\mathrm{H}$ Medizinprodukte GbR, Münster, Germany).

EEG data analysis was performed using custom scripts written in MATLAB R2017b (The Mathworks, Natick, Massachusetts, USA) and using the EEGLAB 14.1.0b open source toolbox [82]. EEG data was first high-pass filtered [zero-phase Hamming windowed sinc FIR, cutoff frequency $(-6 \mathrm{~dB}) 0.5 \mathrm{~Hz}$, passband edge $1 \mathrm{~Hz}$, transition bandwidth $1 \mathrm{~Hz}$, order 1651] and subsequently low-pass filtered [zero-phase Hamming windowed sinc FIR, cut-off frequency $(-6 \mathrm{~dB}) 45 \mathrm{~Hz}$, passband edge 40 $\mathrm{Hz}$, transition bandwidth $10 \mathrm{~Hz}$, order 167]. Further analysis was performed to seven parieto-occipital EEG electrodes $(\mathrm{Pz}, \mathrm{P} 3 / 4, \mathrm{P} 7 / 8$, and $\mathrm{O} 1 / 2)$ only, since this cortical area is widely used to detect individual alpha frequency (IAF) reliably [83, 84]. Channel rejection was performed using the automatic procedure supplied by the clean rawdata EEGLAB extension by taking into account if the correlation of a channel to a reconstruction of it based on other channels, in a given time window, was less than 0.4 as well as if a channel was flat for more than 5 seconds. On average, $\sim 95 \%$ of the parietooccipital channels in the pre-measurement EEG recordings remained for further analysis $(\sigma: \sim 10 \%$; range: $71-100 \%$ ) and $~ 92 \%$ ( $\%: \sim 9 \%$; range: $\sim 71-100 \%$ ) in the post-measurement EEG recordings. Artefactual data points were rejected if their amplitude was higher than \pm $75 \mu \mathrm{V}$ within a $500 \mathrm{~ms}$ width time window as detected by the trimOutlier EEGLAB plugin. On average, 6\% of data was rejected in the pre-measurement EEG recordings ( $\sigma: \sim 9 \%$; range: $\sim 0-30 \%)$ and $\sim 8 \%(\sigma: \sim 14 \%$; range: $\sim 0-48 \%$ ) in the post-measurement EEG recordings. Afterwards, two IAF measures were estimated: peak alpha frequency (PAF) and center of gravity $(\mathrm{CoG})$, by means of the restingIAF v1.0 open source package available from https://github.com/corcorana/restingIAF. This allowed a fully automatic and reliable strategy to determine IAF estimates during resting state EEG recordings, of which a more detailed and extensive description can 
be found elsewhere [84, 85]. Briefly, one-sided channelwise power spectral density (PSD) was first calculated in the $1-40 \mathrm{~Hz}$ frequency range by the Welch's modified periodogram method, using a 2048 sample ( $4 \mathrm{~s})$ Hamming window (50\% overlap) across segments (frequency resolution $=0.244 \mathrm{~Hz}$ ) and normalized by dividing each PSD channel estimate (within the passband) by the mean spectral power. Then, each PSD estimate was smoothed using a Savitzky-Golay filter with frame length equals to 11 frequency bins and polynomial degree of five. From the smoothed PSD and within an a priori defined alpha frequency band $(7-13 \mathrm{~Hz})$, evident frequency peaks were detected and IAF estimates from spectral peaks' boundaries were computed. Using the first derivative to detect spectral peaks seemingly yields true estimates compared to simply searching from maximal values within a predefined alpha frequency band [83]. Finally, IAF estimates were computed by averaging the obtained spectral peaks estimates across channels. The minimum number of valid channels necessary to estimate IAF was set to one, given the relatively low-density parieto-occipital EEG channels used for this analysis. Additionally, spectral power within the alpha frequency band was calculated by averaging in each participant the PSD estimates of all the included channels, and then summing the obtained channels mean power across the alpha frequency band. Alpha spectral bandwidth was defined as the individual PAF $\pm 2 \mathrm{~Hz}$.

\section{Other outcome measures}

The participants wore a StepWatch (Orthocare Innovations LLC, Edmonds, Washington, USA) for 1 week before the intervention period started to assess their daily physical activity behaviour. The StepWatch recorded every step and calculated the number of steps for each day. The mean of 7 days was used as baseline characteristic. Furthermore, the participants rated their current training motivation on a Visual Analog Scale ( $1=$ unmotivated lethargic smiley to $5=$ motivated happy smiley) before every training. After each training session, the participants estimated their perceived exertion on the Borg scale from 6 to 20 ( 6 = "less than very light", 20 = "more than very hard") for Tai Chi-inspired and dance exercises, respectively.

\section{Statistical analysis}

For all statistical analysis, SPSS 23.0 for Windows (SPSS Inc., Chicago, Illinois, USA) was used. Descriptive statistics were generated for all variables. Following a conservative approach and due to non-normality of some of the data, confirmed by both Shapiro-Wilk test and Q-Qplots, non-parametric testing was applied. Intragroup differences between the two measurements were analysed by Wilcoxon signed-rank test. A significance level of $\alpha=0.05$ was applied. Correlational effect sizes (r), according to the following equation: $r=z / \sqrt{ }(n 1+n 2)$ with $\mathrm{n} 1=\mathrm{n}$ at pre-measurement and $\mathrm{n} 2=\mathrm{n}$ at postmeasurement, were calculated in MS Office Excel (version 2016) and reported according to Cohen [86]: an effect size of $r=0.10$ indicates a small effect, $r=0.30$ a medium effect, and $r \geq 0.50$ a large effect. For pre- and post-measurement comparisons, drop-outs were excluded from analysis (per-protocol analysis). The analysis does not consider intention-to-treat analysis because of a clear description of the drop-out reasons [87]. Moreover, only participants who reached $70 \%$ of the maximal possible training sessions were included in the pre-post-comparison.

\section{Results}

A total of 21 participants signed informed consent and were included in the study. Nineteen participants completed the seven-week training intervention. The two male, 71 years old and highly educated drop-outs were comparable to the rest of the sample regarding their characteristics. Table 1 summarizes the demographic characteristics and screening measures of the remaining participants. The study flow chart is presented in Fig. 2.

\section{Primary outcome results}

The scores of the SUS and GEQ measured after the three initial training sessions (between-measurement) and after the training period (post-measurement) are presented in Table 2. The additional question, asking participants about their general opinion of the Active@Home exergame, showed a mean score $( \pm \mathrm{SD})$ of $3.1 \pm$ $0.7(n=20)$ at between-measurement and a mean score $( \pm \mathrm{SD})$ of $3.0 \pm 0.8(n=19)$ at post-measurement (on a scale from $0=$ "I don't like it" to $4=$ "I like it a lot"). Table 3 summarizes the main feedback of the participants and observations of the supervisors during the training. No adverse events were recorded during the intervention period.

Participants completed on average 18 out of the 21 total training sessions, resulting in an adherence rate of 90.0\%. Six participants reached the recommended training dose of three training sessions per week. Reasons for non-adherence were: holidays, being busy and family affairs. Considering the two drop-outs, the attrition rate amounted to $9.5 \%$. One drop-out reason was related to a lack of training motivation and the other reason was related to personal issues.

\section{Secondary outcome results}

On group level, the gait speed under the dual-task walking condition increased significantly $(\mathrm{z}=-2.012$, $p=.045, \mathrm{r}=0.33)$ after the training intervention. In the 5 -chair rises test of the SPPB, the time needed to perform five chair rises significantly decreased $(\mathrm{z}=-2.853$, $p=.003, \mathrm{r}=0.46)$ after exergame training. In the divided 
Table 1 Demographic characteristics and screeing values

\begin{tabular}{|c|c|}
\hline Participant characteristics & $n=19$ \\
\hline Age in years & $71.4 \pm 6.1(65-91)$ \\
\hline Weight [kg] & $69.7 \pm 19.5(42-122)$ \\
\hline Height $[\mathrm{cm}]$ & $169.9 \pm 8.8(150-181)$ \\
\hline BMI $\left[\mathrm{kg} / \mathrm{m}^{2}\right]$ & $24.3 \pm 5.0(17-37)$ \\
\hline Daily physical activity ${ }^{a}$ & $7410 \pm 2079(4605-12,247)$ \\
\hline MOCA Score & $28.1 \pm 1.4(26-30)$ \\
\hline Female $[\mathrm{n}, \%]$ & $10(52.6)$ \\
\hline \multicolumn{2}{|l|}{ Education [n, \%] } \\
\hline Primary school & $1(5.3)$ \\
\hline Upper school & $0(0.0)$ \\
\hline Apprenticeship & $9(47.4)$ \\
\hline Gymnasium & $2(10.5)$ \\
\hline University & $7(36.8)$ \\
\hline \multicolumn{2}{|l|}{ Fear of falling [n, \%] } \\
\hline Never & $14(73.7)$ \\
\hline Sometimes & $5(26.3)$ \\
\hline Often & $0(0.0)$ \\
\hline Always & $0(0.0)$ \\
\hline \multicolumn{2}{|c|}{ Number of falls during last month ${ }^{b}[n, \%]$} \\
\hline Never & $17(89.5)$ \\
\hline Once & $2(10.5)$ \\
\hline More than once & $0(0.0)$ \\
\hline \multicolumn{2}{|c|}{ Self-evaluation of health state [n, \%] } \\
\hline Very good & $4(21.1)$ \\
\hline Good & $14(73.7)$ \\
\hline Medium & $1(5.3)$ \\
\hline Bad & $0(0.0)$ \\
\hline \multicolumn{2}{|c|}{ Self-evaluation of balance [n, \%] } \\
\hline Very good & $5(26.3)$ \\
\hline Good & $8(42.1)$ \\
\hline Medium & $6(31.6)$ \\
\hline Bad & $0(0.0)$ \\
\hline \multicolumn{2}{|c|}{ Self-evaluation of muscle strength [n, \%] } \\
\hline Very good & $0(0.0)$ \\
\hline Good & $18(94.7)$ \\
\hline Medium & $0(0.0)$ \\
\hline Bad & $1(5.3)$ \\
\hline
\end{tabular}

Data are mean values \pm standard deviation (range) or number of participants per category (absolute and relative frequency) as indicated. MoCA Montreal

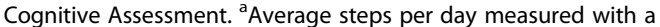
StepWatch. ${ }^{b}$ Self-stated.

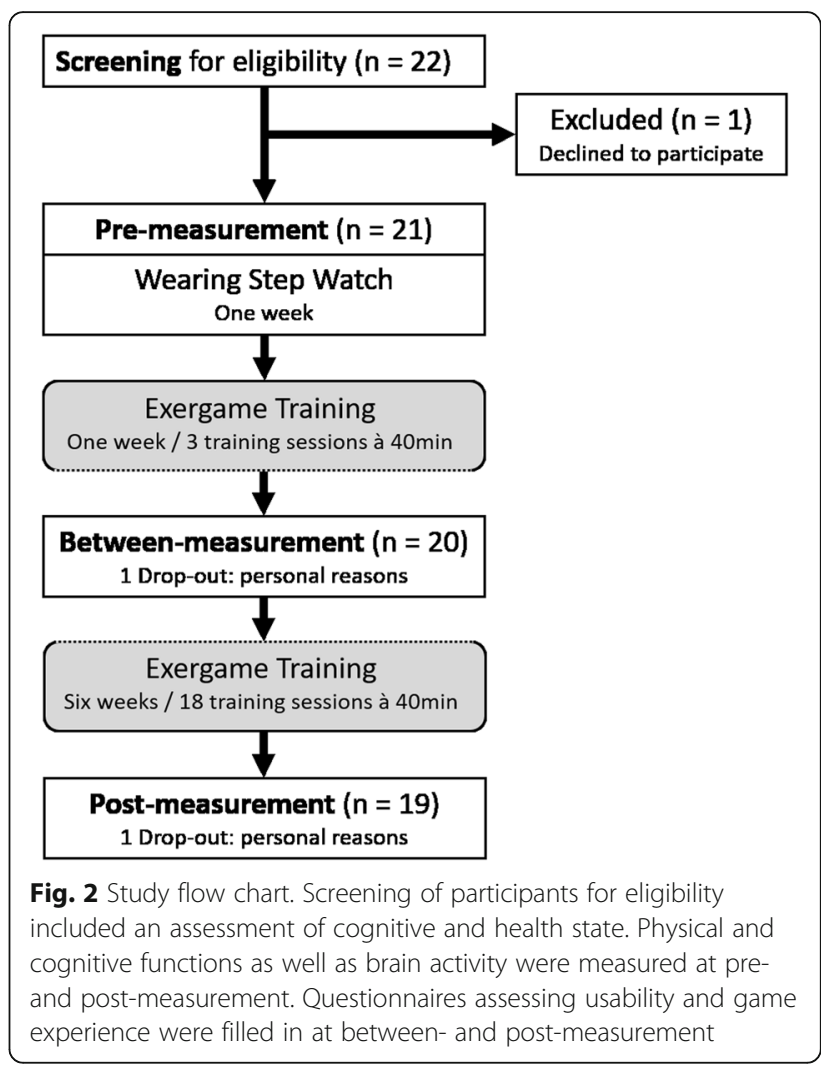

attention task of the TAP, participants reacted significantly faster $(\mathrm{z}=-2.495, p=.011, \mathrm{r}=0.40)$ to auditory stimuli after the training intervention. No significant changes in resting state EEG were found for the preand post-measurement comparison. The results are presented in Table 4.

\section{Other outcome results}

The participants' average daily physical activity resulted in 7410 steps per day (Table 1). Training motivation was on average rated with $4.5 \pm 0.5$ by participants (on a visual scale from $1=$ unmotivated lethargic smiley to $5=$ motivated happy smiley). The average rating of participants' perceived exertion for Tai Chi-inspired exercises was $10.7 \pm 2.3$ and for dance exercises $9.7 \pm 2.0$ on the 20 -point Borg scale $(6=$ "less than very light" to $20=$ "more than very hard").

\section{Discussion}

The primary aim of this study was to assess the usability of the newly developed Active@Home exergame prototype in older adults and to provide implications for further development. Furthermore, potential training-related changes in physical and cognitive functioning were explored. In general, the newly developed exergame seems usable for healthy older adults and the study results indicate possible 
Table 2 Results of questionnaire ratings

\begin{tabular}{|c|c|c|c|c|c|}
\hline Questionnaires & $\mathrm{T} 2(n=20)$ & $\mathrm{T} 3(n=19)$ & z & $p$ & r \\
\hline System Usability Scale (SUS) & $75.0(67.5 ; 87.5)$ & $75.0(70.0 ; 85.0)$ & -0.240 & .823 & 0.04 \\
\hline \multicolumn{6}{|c|}{ Game Experience Quesionnaire (GEQ) } \\
\hline Competence & $2.5(2.2 ; 2.7)$ & $2.3(2.2 ; 3.0)$ & -0.081 & .945 & 0.01 \\
\hline Immersion & $1.9(1.5 ; 2.5)$ & $2.3(1.5 ; 2.7)$ & -0.881 & .395 & 0.14 \\
\hline Flow & $1.3(0.7 ; 1.8)$ & $1.0(0.7 ; 1.5)$ & -1.281 & .210 & 0.21 \\
\hline Tension & $0.2(0.0 ; 0.5)$ & $0.2(0.0 ; 0.3)$ & -1.279 & .229 & 0.21 \\
\hline Challenge & $1.2(1.0 ; 1.5)$ & $1.2(0.7 ; 1.5)$ & -1.455 & .157 & 0.24 \\
\hline Negative Affect & $0.2(0.0 ; 0.6)$ & $0.5(0.2 ; 0.8)$ & -3.134 & $.001^{*}$ & 0.51 \\
\hline Positive Affect & $2.8(2.4 ; 3.2)$ & $2.8(2.0 ; 3.3)$ & -0.569 & .590 & 0.09 \\
\hline
\end{tabular}

Data are median values (interquartile range). T2 = after three training sessions (between-measuement), T3=after training intervention (post-measurement). ${ }^{*} p<.05, p$-values are exact sig. Two-tailed. T2-T3 differences were evaluated using Wilcoxon signed-rank test $(n=19)$. An effect size of $r=0.10$ indicates a small effect, $r=0.30$ a medium effect, and $r \geq 0.50$ a large effect [86]. SUS scale ranges from 0 to 100 . GEQ scale ranges from $0=$ "not at all" to $4=$ "extremly". Two categories of the GEQ (tension, negative affect) have to be evaluated reversely which means a low score is favorable.

positive influences on physical and cognitive functions. However, the later have to be interpreted with caution due to the lack of a control group.

\section{Usability of the exergame training}

An acceptable usability of the Active@Home exergame prototype was evident in the questionnaire ratings with a SUS score of $75 / 100$ after three training sessions as well as at the end of the intervention. This result is in line with previous studies showing that exergames are in general well accepted and usable for older adults, especially when considering their needs $[29,35,64]$. However, some limitations were evident in the observations of supervisors during training and in the feedback of participants. Most limitations were related to technical issues that occurred during training with the exergame prototpye (e.g. system crashes or unstable connection to IMUs). Considering that older adults' technical knowledge and experiences with new technologies are often restricted, a newly developed technology-based system has to work without any technical failures. Moreover, a simple set-up, stable connections, and an intuitive interaction with the system are mandatory aspects especially when the training system is expected to be deployed at older adults' home. The importance of an age-appropriate design and flawless technical functionality were emphasized to be crucial for the usability of exergames $[64,88,89]$. Otherwise, technological problems can lead to unintentional avoidance and disuse of a training system.

Even though some technical issues occurred during training with the exergame prototype, training compliance in this study was high. The adherence rate of $90.0 \%$ indicates a good acceptance of the training system. Moreover, an attrition rate of less than $10 \%$ (9.5\% in this study) can be considered acceptable. Interestingly, the reasons for non-adherence were not related to the usability of the exergame. Only one participant prematurely terminated the intervention due to a lack of motivation. However, the general training motivation was rated high (on average 4.5/5). High motivation is an important key factor for long-term exercising that, in turn, leads to lasting training effects [90,91]. In accordance with our results, adherence has been shown to be often higher in interventions using exergames compared to standard fall prevention exercises $[36,69,92]$. An explanation might be the entertaining and captivating character of exergames resulting in positive emotions [64, 66, 93]. Positive emotional experiences, in turn, might increase training motivation and therefore, as mentioned before, enhance long-term compliance [94]. Regarding emotions in this study, the GEQ scores showed medium to high ratings of positive emotions, e.g. feeling captivated and pleased, as well as low ratings of negative emotions, e.g. feeling tensed and annoyed, both after three training sessions and at the end of the intervention. The significant increase in negative affect from between- to post-measurement might be due to an accumulation of negative emotions related to technical issues. Nevertheless, we can conclude that the Active@Home exergame provided an overall positive emotional experience which might have led to the high training motivation and the high training compliance found in this study.

In order to maximize the benefits of a training, exercise interventions should implement some basic training principles including feedback, optimal load, progression and variability [57]. The supervisors' observations and participants' statements showed that the feedback system integrated in the Active@Home exergame was, currently, not yet optimal. To facilitate learning and the acquisition of new skills and knowledge, optimal feedback is mandatory [95]. Negative feedback helps to correct errors while positive feedback satisfies and often increases motivation [95]. One reason for the dissatisfaction with the feedback system might be that the movement evaluation was slightly inaccurate and, thus, the evaluation 
Table 3 Summary of usability protocol with supervisors' observations and participants' feedback

\begin{tabular}{|c|c|c|}
\hline Criteria & Positive aspects & Negative aspects \\
\hline \multirow{4}{*}{$\begin{array}{l}\text { Functionality and } \\
\text { interaction with the } \\
\text { system }\end{array}$} & $\begin{array}{l}\text { - Good and stable connection of laptop (with system } \\
\text { software) to TV }\end{array}$ & $\begin{array}{l}\text { - Technical issues (as system crashes or frozen pictures on } \\
\text { the screen) }\end{array}$ \\
\hline & - Simple set up ${ }^{\mathrm{b}}$ & - Unstable (Bluetooth) connection of IMUs to the system ${ }^{\mathrm{a}, \mathrm{b}}$ \\
\hline & - Easy usable game composition ${ }^{\mathrm{b}}$ & $\begin{array}{l}\text { - Navigation via laptop keyboard instead by IMUs (as } \\
\text { cursors) }^{\mathrm{a}}\end{array}$ \\
\hline & & $\begin{array}{l}\text { - Inaccurate evaluation of movements by IMUs (evaluation } \\
\text { algorithms) }\end{array}$ \\
\hline \multirow[t]{3}{*}{ IMUs } & $\begin{array}{l}\text { - Comfortable to wear (participants did not notice them } \\
\text { during training) }\end{array}$ & $\begin{array}{l}\text { - Suboptimal material of IMU cover (cover expanded after } \\
\text { heating up while charging) }\end{array}$ \\
\hline & & $\begin{array}{l}\text { - Suboptimal material of IMU fixation strap (difficult to clean } \\
\text { the Velcro fixation, material sticks to some clothes) }{ }^{a, b}\end{array}$ \\
\hline & & $\begin{array}{l}\text { - Difficulties to attach the IMUs with the Velcro fixation } \\
\text { (especially at wrists) }^{a, b}\end{array}$ \\
\hline \multirow[t]{5}{*}{ Design } & $\begin{array}{l}\text { - Exciting game story of travelling around Europe to } \\
\text { different cities }\end{array}$ & - No variation in music ${ }^{b}$ \\
\hline & - Pleasant music ${ }^{b}$ & $\begin{array}{l}\text { - No explanation about feedback system (colour code, } \\
\text { performance score) }\end{array}$ \\
\hline & - Helpful cues (arrows) to prepare the next movement ${ }^{b}$ & \\
\hline & - Virtual instructor guiding through exercises ${ }^{\mathrm{b}}$ & \\
\hline & - Helpful indication of number of exercise repetitions ${ }^{\mathrm{b}}$ & \\
\hline \multirow[t]{4}{*}{ Training principles } & $\begin{array}{l}\text { - Visual feedback with colour code (green, orange, red) } \\
\text { during exercising }\end{array}$ & $\begin{array}{l}\text { - No specific feedback regarding exercise execution and } \\
\text { single body part movements }{ }^{a, b}\end{array}$ \\
\hline & - Performance score as feedback after exercising ${ }^{b}$ & $\begin{array}{l}\text { - Training load and progression determined by supervisors } \\
\text { (no automatic progression) }\end{array}$ \\
\hline & & - Low variability in exercises ${ }^{\mathrm{a}, \mathrm{b}}$ \\
\hline & & - Training load even in high levels not exhausting ${ }^{1,2}$ \\
\hline \multirow[t]{3}{*}{ Exercises } & - Clear structure of exercise levels ${ }^{b}$ & $\begin{array}{l}\text { - No proper introduction of exercises (just start copying the } \\
\text { movements of the virtual instructor) }\end{array}$ \\
\hline & $\begin{array}{l}\text { - Complex exercises with additional arm movements } \\
\text { provide more fun than simple (boring) movements }{ }^{b}\end{array}$ & - Only frontal view of exercises (side view missing) ${ }^{\mathrm{b}}$ \\
\hline & & $\begin{array}{l}\text { - No further information about exercise (e.g. muscles } \\
\text { involved) }\end{array}$ \\
\hline \multirow[t]{3}{*}{ Emotions } & - General enjoyment and fun ${ }^{\mathrm{a}, \mathrm{b}}$ & $\begin{array}{l}\text { - Frustrated and displeased by technical issues and } \\
\text { inaccurate evaluation of movements }{ }^{a, b}\end{array}$ \\
\hline & $\begin{array}{l}\text { - Increased motivation through virtual instructor (better } \\
\text { than train alone) }\end{array}$ & - Missing challenge due to easy exercises ${ }^{a, b}$ \\
\hline & $\begin{array}{l}\text { - Happy when seeing a progress or achieving higher } \\
\text { performance score }{ }^{a, b}\end{array}$ & - Bored of low training variability ${ }^{\mathrm{a}, \mathrm{b}}$ \\
\hline
\end{tabular}

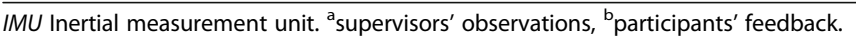

algorithms have to be improved. Another reason might be that the implemented feedback system was too superficial (e.g. evaluation of whole-body movements), while more specific and precise feedback was missing (e.g. feedback of upper/lower limbs' movements separately). Nevertheless, participants could benefit from the real-time feedback while training and were proud and motivated when reaching high performance scores. Considering the training principles of optimal load and progression, the exercise demands have to be continuously adapted to each participant's skill level achieving a challenging situation and avoiding an under- or overload. For the Active@Home exergame intervention, however, some participants stated that the training was not challenging enough. A reason might be that the exergame had to comply with a wide range of ability levels of older adults. Therefore, more difficult and complex exercises should be added to the exergame to satisfy also fitter older adults. Despite these limitations, participants generally enjoyed the simple system set-up, the game story of travelling around Europe, the music during the exercises and the guidance of the virtual instructor. 
Table 4 Results of pre- and post-measurements of physical and cognitive functions and cortical acitivty

\begin{tabular}{|c|c|c|c|c|c|c|}
\hline & Assessed by & Pre (T1) & Post (T3) & z & $\mathrm{p}$ & $r$ \\
\hline \multirow[t]{24}{*}{ Physical functions } & Gait analysis & & & & & \\
\hline & Speed $[\mathrm{m} / \mathrm{s}]$ & & & & & \\
\hline & ST walking & $1.42(1.36 ; 1.61)$ & $1.52(1.36 ; 1.62)$ & -0.684 & .515 & 0.11 \\
\hline & DT walking & $1.26(1.17 ; 1.46)$ & $1.31(1.22 ; 1.51)$ & -2.012 & $.045^{*}$ & 0.33 \\
\hline & DT costs in \% & $6.4(5.1 ; 17.6)$ & $8.4(3.0 ; 18.2)$ & -1.449 & .156 & 0.24 \\
\hline & Cadence $[$ steps/min] & & & & & \\
\hline & ST walking & $117.2(114.6 ;$ & $120.7(115.1 ; 126.1)$ & -1.529 & .134 & 0.25 \\
\hline & DT walking & 125.7) & $113.2(109.5 ; 120.4)$ & -1.690 & .096 & 0.27 \\
\hline & DT costs in \% & $\begin{array}{l}111.7(105.4 ; 116.9) \\
4.0(1.8 ; 8.7)\end{array}$ & $3.5(1.0 ; 9.0)$ & -1.046 & .312 & 0.17 \\
\hline & Stride length $[\mathrm{m}]$ & & & & & \\
\hline & ST walking & $1.48(1.41 ; 1.56)$ & $1.47(1.41 ; 1.56)$ & -0.402 & .709 & 0.07 \\
\hline & DT walking & $1.36(1.28 ; 1.50)$ & $1.36(1.33 ; 1.49)$ & -1.610 & .113 & 0.26 \\
\hline & DT costs in \% & $3.8(2.1 ; 10.3)$ & $4.2(0.0 ; 9.2)$ & -1.006 & .332 & 0.16 \\
\hline & Minimal toe clearance $[\mathrm{cm}]$ & & & & & \\
\hline & ST walking & $2.3(2.1 ; 3.1)$ & $2.9(2.0 ; 3.4)$ & -1.891 & .060 & 0.31 \\
\hline & DT walking & $2.2(1.7 ; 2.7)$ & $2.7(1.6 ; 3.2)$ & -1.248 & .225 & 0.20 \\
\hline & DT costs in \% & $3.9(-4.9 ; 16.6)$ & $6.7(-7.1 ; 19.3)$ & -0.241 & .829 & 0.04 \\
\hline & Extended SPPB & & & & & \\
\hline & Total score & $14(13 ; 15)$ & $14(13 ; 15)$ & -0.266 & .797 & 0.04 \\
\hline & Balance score & $6(6 ; 8)$ & $7(5 ; 7)$ & -0.134 & .947 & 0.02 \\
\hline & 4 m-gait score & $4(4 ; 4)$ & $4(4 ; 4)$ & 0.000 & 1.000 & $<0.01$ \\
\hline & $4 \mathrm{~m}$-gait time $[\mathrm{s}]$ & $3.3(2.9 ; 3.7)$ & $3.2(2.8 ; 3.6)$ & -1.449 & .153 & 0.24 \\
\hline & 5-chair rises score & $4(3 ; 4)$ & $4(3 ; 4)$ & -0.816 & .750 & 0.13 \\
\hline & 5-chair rises time $[s]$ & $10.5(8.3 ; 12.8)$ & $8.8(7.3 ; 12.3)$ & -2.853 & $.003^{*}$ & 0.46 \\
\hline \multirow[t]{17}{*}{ Cognitive functions } & Test of Attentional Performance & & & & & \\
\hline & Working memory & & & & & \\
\hline & RT [ms] & $741(597 ; 843)$ & $677(603 ; 840)$ & -0.348 & .742 & 0.06 \\
\hline & Errors & $3(0 ; 6)$ & $3(1 ; 5)$ & -0.416 & 696 & 0.07 \\
\hline & Omissions & $3(2 ; 4)$ & $3(1 ; 4)$ & -1.719 & .088 & 0.28 \\
\hline & Divided attention & & & & & \\
\hline & RT auditory [ms] & $652(584 ; 769)$ & $594(580 ; 714)$ & -2.495 & $.011^{*}$ & 0.40 \\
\hline & RT visual [ms] & $893(822 ; 948)$ & $881(834 ; 945)$ & -0.080 & .945 & 0.01 \\
\hline & Errors & $1(0 ; 3)$ & $1(0 ; 2)$ & -0.641 & .541 & 0.10 \\
\hline & Omissions & $1(0 ; 3)$ & $1(1 ; 2)$ & -1.388 & .190 & 0.23 \\
\hline & Selective attention & & & & & \\
\hline & RT [ms] & $454(397 ; 487)$ & $468(396 ; 504)$ & -0.543 & .602 & 0.09 \\
\hline & Errors & $1(0 ; 2)$ & $0(0 ; 2)$ & -0.265 & .848 & 0.04 \\
\hline & Omissions & $0(0 ; 0)$ & $0(0 ; 0)$ & -1.890 & .125 & 0.31 \\
\hline & Mental flexibility & & & & & \\
\hline & $\mathrm{RT}[\mathrm{ms}]$ & $932(798 ; 1124)$ & $848(786 ; 1018)$ & -1.610 & .113 & 0.26 \\
\hline & Errors & $3(1 ; 9)$ & $3(1 ; 4)$ & -1.163 & .258 & 0.19 \\
\hline \multirow[t]{2}{*}{ Cortical activity } & Resting state EEG & & & & & \\
\hline & Peak alpha frequency $[\mathrm{Hz}]$ & $9.3(8.4 ; 9.9)$ & $9.3(8.7 ; 10.0)$ & -1.274 & .232 & 0.26 \\
\hline
\end{tabular}


Table 4 Results of pre- and post-measurements of physical and cognitive functions and cortical acitivty (Continued)



Data are median values (interquartile range). $\mathrm{n}=19$. ${ }^{*} \mathrm{p}<.05, \mathrm{p}$-values are exact sig. Two-tailed. Pre-post differences were evaluated using Wilcoxon signed-rank test. An effect size of $r=0.10$ indicates a small effect, $r=0.30$ a medium effect, and $r \geq 0.50$ a large effect [86]. ST single-task, DT dual-task. DT costs are calculated as (ST $-\mathrm{DT}) / \mathrm{ST} \times 100$. SPPB Short Physical Performance Battery. $R T$ reaction time

To summarize, this study showed a useable exergame prototype with several points that should be considered for further improvement of the system: technical aspects, movement evaluation and implementation of training principles. After this first evaluation of the newly developed exergame prototype in a monitored and supervised environment, the findings warrant an extended trial testing the feasibility and usability of the adjusted Active@Home exergame in an in-home setting.

\section{Exploration of potential effects of the exergame training} Our study showed significant improvement of dual-task walking speed after exergame training, which is in line with the results of several other studies [74, 96, 97]. Exergaming is discussed to train dual-task abilities, which are important in daily life activities and for fall prevention in older adults [98]. Furthermore, pre-post comparisons revealed a significant increase of functional lower limbs muscle power in this study. This improvement might be related to the Tai Chi-inspired exercises embedded in the exergame training. These exercises were mainly performed in squat position placing load on the lower limb and core muscles [99-101]. Despite of this potential improvement in muscle strength, the training guidelines considered for strength training turned out to be unsuitable [44]: The required intensity of $70-79 \%$ of 1 RM (one repetition maximum) could not be reached using mainly body weight-based exercises. Furthermore, the breaks in between strength exercises, composed for very large exercise efforts, were too long. Consequently, the participants' subjective rating of perceived exertion for Tai Chiinspired training was on average on a low rate (10.7/20). The optimal zone for strength training corresponds to Borg scale ratings of $15-17$ on the 20-point scale [102, 103]. The suboptimal training load and the rather short training period of 7 weeks might have restricted the training impact on additional physical outcomes. We, therefore, recommend to increase exercise complexity and intensity and extend the training period.

In the computer-based cognitive tests, study results showed a significantly improved reaction time for auditory stimuli in a divided attention task in pre-post comparisons. Surprisingly, the reaction time to visual stimuli did not improve. This could be related to the fact that especially for dance exercises listening to auditory information (music, rhythms) was important. These findings are similar to the results of a recent exergame study in older adults [74]. Furthermore, several studies including a combined physical-cognitive training as exergaming provided indications that this training approach boosts particular executive functions as mental flexibility, inhibition, or working memory [96, 104-106]. Executive functions are higher order cognitive functions playing an important role in guiding through everyday life. The absence of improvements in executive functions in this study might be due to the fact that the cognitive training itself was not specific enough or too short.

For older adults, a variety of studies reported agerelated changes in cortical oscillatory activity [107]. Most of these studies showed a general "slowing" of the resting state EEG with a power increase in the slow frequency ranges $(<7 \mathrm{~Hz})$ and a power decrease in higher frequencies (e.g. alpha power frequency band: 7-13 Hz) especially in posterior brain regions [13, 14]. Accordingly, the individual alpha frequency peak is known to decrease in the later part of lifespan [107]. Furthermore, alpha power is considered to be positively correlated to global cognitive status in healthy and impaired older adults [107-110]. The pathological processes on neuronal level during aging leading to altered EEG power are presumed to be counteracted with physical and cognitive training [14]. However, we found no significant changes in resting state EEG in pre-post comparisons in our study, which might be also due to the short intervention period.

\section{Limitations}

As this is a pilot study with primary focus on usability outcomes, secondary results have to be interpreted with caution especially because of small sample size and missing control group [111, 112]. To carefully evaluate the effectiveness of the Active@Home exergame, a further larger study (randomized controlled trial) will be conducted. Moreover, the training period should be extended to increase the potential for training improvements. Regarding the relatively high physical fitness level of the participants, caution has to be provided by generalizing these results to the overall older adults. To ensure high acceptability, newly developed training systems and interventions must be developed according to the target users' needs, attitudes, and expectations. One reason for the abovementioned result in this study of general high usability might 
be that the development and design of the Active@Home exergame has followed a user-centered design approach. Older adults as target users have been involved from the beginning in the processes of training and game development. Nevertheless, a discrepancy exists between the high usability ratings in the questionnaires compared to the limitations observed by the supervisors and stated by the participants during training. This "positive bias" in the quantitative methods might be due to a general enthusiasm of the participants for the basic idea of the training approach, their pleasure to exercise despite of the potential for improvement of the training system, their satisfaction to support research or due to social aspects.

\section{Implications}

Based on the results of this usability study, several implications can be provided to support the development and improvement of exergame training approaches for older adults:

- A mature concept should be composed including game design, technology and training aspects.

- The system set-up should be simple and ageappropriate (e.g. regarding technological devices, screen size, game design, in-program navigation).

- If technological devices as IMUs are attached to players' bodies, material and fixation must be optimized, user-friendly, comfortable and sizeadjustable.

- Easy applicable charging solutions should be used for technological devices as IMUs.

- Players should be sufficiently informed about the game story, goals, evaluation and feedback system.

- Exercises have to be well explained and instructed before and during their execution (e.g. exercise tutorial with frontal and side view, further information about exercise goals, cues during execution, and time indications).

- Including music in exergames might be important and motivating provided that the music is appropriate and fits the game story, training content and movements.

- Performance feedback has to be accurate, easy to understand and as detailed as possible, since feedback is one of the most important motivational factors and necessary for training benefits.

- Games and exercises should be challenging for a wide range of player prerequisites.

- Automatic configuration of optimal training load and progression is desirable.

- A high variability in games and exercise options is needed.

\section{Conclusion}

Our study results showed a general high usability of the newly developed Active@Home exergame with high training compliance and positive emotional game experience in older adults. The results of this study provide some important suggestions for further exergame development and other technology-based training systems for older adults. Implications include elimination of technical issues as well as ensuring a simple system set-up, accurate feedback and challenging and diverse exercises for a wide range of skill levels. Furthermore, the study results provide some indications that this multicomponent video game-based training might enhance physical and cognitive functions in older adults. The study results warrant further development of the Active@Home exergame and may help other researchers in the design process of exergame interventions for older adults.

\section{Abbreviations \\ DT: Dual-task; DTC: Dual-task cost(s); EEG: Electroencephalography; GEQ : Game experience Questionnaire; IMU : Inertial Measurement Unit; MOCA: Montreal Cognitive Assessment; SPPB: Short Physical Performance Battery; ST: Single-task; SUS: System Usability Scale; TAP: Test of Attentional Performance}

\section{Acknowledgments \\ The authors would like to thank Melanie Thalmann and Laura Walker for supporting testing and training. Furthermore, we would like to thank all participants for their kindness and patience during the testing and training sessions. Moreover, the authors would like to thank also all the national and international partners involved in the Active@Home project and exergame development.}

\section{Authors' contributions}

MA and FS developed the research question under the lead of EDB. The concept and design were established by MA and FS while AS and EDB acted as methodological council. MA and FS conducted data acquisition, analysis and interpretation of the results with edition and improvement by AS and EDB. FG performed EEG data analysis and interpretation of the EEG results which was edited and improved by MA, AS and EDB. MA and FS produced a first version of the manuscript. AS, FG and EDB substantially revised the manuscript to bring it to its current version. All authors have read and approved the final manuscript.

\section{Funding}

This work was partially supported by the AAL Active@Home project (www. aal-europe.eu/projects/activehome/), funded by the European Commission and co-funded by the Swiss Confederation represented by the State Secretariat for Education, Research and Innovation (SERl; agreement number 1315000595).

Availability of data and materials

All data generated and analysed during this study are included in this published article.

Ethics approval and consent to participate

The ETH Zurich Ethics Committee (Zurich, Switzerland) granted ethical approval (protocol number EK 2017-N-06). All participants were fully informed prior to participation and signed an informed consent form according to the Declaration of Helsinki before conducting any measurement.

Consent for publication

Institutional consent form signed by the study participant in Fig. 1 is available on request. 


\section{Competing interests}

The authors declare that they have no competing interests.

\section{Author details}

'Department of Health Sciences and Technology, Institute of Human Movement Sciences and Sport, ETH Zurich, Leopold-Ruzicka-Weg 4, 8093 Zurich, Switzerland. 'Department of Neurobiology, Care Sciences and Society, Karolinska Institute, Alfred Nobels Alle 23, 14183 Huddinge, Sweden.

\section{Received: 13 June 2019 Accepted: 19 December 2019}

\section{Published online: 11 January 2020}

\section{References}

1. Willett, W.C., et al., Prevention of chronic disease by means of diet and lifestyle changes. 2006.

2. Berg WP, et al. Circumstances and consequences of falls in independent community-dwelling older adults. Age Ageing. 1997;26(4):261-8.

3. Hausdorff JM, Rios DA, Edelberg HK. Gait variability and fall risk in community-living older adults: a 1-year prospective study. Arch Phys Med Rehabil. 2001:82(8):1050-6.

4. Tinetti ME, Speechley M, Ginter SF. Risk factors for falls among elderly persons living in the community. N Engl J Med. 1988;319(26):1701-7.

5. Taylor ME, et al. Gait impairment and falls in cognitively impaired older adults: an explanatory model of sensorimotor and neuropsychological mediators. Age Ageing. 2012;41(5):665-9.

6. Rubenstein LZ. Falls in older people: epidemiology, risk factors and strategies for prevention. Age Ageing. 2006;35(suppl 2):ii37-41.

7. Schoene $D$, et al. The effect of interactive cognitive-motor training in reducing fall risk in older people: a systematic review. BMC Geriatr. 2014;14(1):1.

8. Skelton DA, Dinan SM. Exercise for falls management: rationale for an exercise programme aimed at reducing postural instability. Physiother Theory Pract. 1999;15(2):105-20.

9. Sherrington $C$, et al. Exercise to prevent falls in older adults: an updated meta-analysis and best practice recommendations. N S W Public Health Bull. 2011;22(4):78-83.

10. Raz N, et al. Regional brain changes in aging healthy adults: general trends, individual differences and modifiers. Cereb Cortex. 2005;15(11):1676-89.

11. Resnick SM, et al. Longitudinal magnetic resonance imaging studies of older adults: a shrinking brain. J Neurosci. 2003;23(8):3295-301.

12. Gunning-Dixon FM, et al. Aging of cerebral white matter: a review of MRI findings. Int J Geriatr Psychiatry. 2009;24(2):109-17.

13. Rossini PM, et al. Clinical neurophysiology of aging brain: from norma aging to neurodegeneration. Prog Neurobiol. 2007;83(6):375-400.

14. Babiloni $C$, et al. Brain neural synchronization and functional coupling in Alzheimer's disease as revealed by resting state EEG rhythms. Int J Psychophysiol. 2016;103:88-102.

15. de Bruin E, Schmidt A. Walking behaviour of healthy elderly: attention should be paid. Behav Brain Funct. 2010;6:59-67.

16. Segev-Jacubovski $\mathrm{O}$, et al. The interplay between gait, falls and cognition: can cognitive therapy reduce fall risk? Expert Rev Neurother. 2011;11(7): 1057-75.

17. Yogev-Seligmann G, Hausdorff JM, Giladi N. The role of executive function and attention in gait. Mov Disord. 2008;23(3):329-42.

18. Holtzer $\mathrm{R}$, et al. Cognitive processes related to gait velocity: results from the Einstein aging study. Neuropsychology. 2006;20(2):215

19. Mirelman A, et al. Executive function and falls in older adults: new findings from a five-year prospective study link fall risk to cognition. PLoS One. 2012 7(6):e40297.

20. Delbaere $K$, et al. A multifactorial approach to understanding fall risk in older people. J Am Geriatr Soc. 2010;58(9):1679-85.

21. Hausdorff $J M$, et al. Walking is more like catching than tapping: gait in the elderly as a complex cognitive task. Exp Brain Res. 2005;164(4):541-8.

22. Li KZH, Lindenberger U. Relations between aging sensory/sensorimotor and cognitive functions. Neurosci Biobehav Rev. 2002;26(7):777-83.

23. Pichierri $\mathrm{G}$, et al. Cognitive and cognitive-motor interventions affecting physical functioning: a systematic review. BMC Geriatr. 2011;11:29.

24. Bamidis $P$, et al. A review of physical and cognitive interventions in aging Neurosci Biobehav Rev. 2014:44:206-20.

25. Penedo FJ, Dahn JR. Exercise and well-being: a review of mental and physical health benefits associated with physical activity. Curr Opin Psychiatry. 2005;18(2):189-93.
26. Warburton DE, Nicol CW, Bredin SS. Health benefits of physical activity: the evidence. Can Med Assoc J. 2006:174(6):801-9.

27. Sherrington $C$, et al. Effective exercise for the prevention of falls: a systematic review and meta-analysis. J Am Geriatr Soc. 2008:56(12):2234-43.

28. de Bruin $E$, et al. Use of virtual reality technique for the training of motor control in the elderly some theoretical considerations. Zeitschrift Fur Gerontologie Und Geriatrie. 2010;43(4):229-34

29. Gerling K, Mandryk R. Custom-designed motion-based games for older adults: a review of literature in human-computer interaction. Gerontechnology. 2014;12(2):68-80.

30. Pirovano $\mathrm{M}$, et al. Exergaming and rehabilitation: a methodology for the design of effective and safe therapeutic exergames. Entertainment Comput. 2016:14:55-65.

31. Hoffmann $\mathrm{K}$, et al. Personalized adaptive control of training load in cardioExergames--a feasibility study. Games Health J. 2015:4(6):470-9.

32. Brox E, Konstantinidis ST, Evertsen G. User-centered Design of Serious Games for older adults following 3 years of experience with Exergames for seniors: a study design. JMIR Serious Games. 2017;5(1):e2

33. Ofli $F$, et al. Design and evaluation of an interactive exercise coaching system for older adults: lessons learned. IEEE J Biomed Health Inform. 2016; 20(1):201-12.

34. Campbell M, et al. Framework for design and evaluation of complex interventions to improve health. Br Med J. 2000;321(7262):694-6.

35. Nawaz A, et al. Usability and acceptability of balance exergames in older adults: a scoping review. Health Informatics J. 2016;22(4):911-31.

36. Wuest $\mathrm{S}$, et al. Usability and effects of an Exergame-based balance training program. Games Health J. 2014;3(2):106-14.

37. Macefield R. How to specify the participant group size for usability studies: a practitioner's guide. J Usability Stud. 2009;5(1):34-45.

38. Field T. Tai chi research review. Complement Ther Clin Pract. 2011;17(3):141-6.

39. Persch $L N$, et al. Strength training improves fall-related gait kinematics in the elderly: a randomized controlled trial. Clin Biomech. 2009:24(10):819-25.

40. Aboutorabi A, et al. The effect of aging on gait parameters in able-bodied older subjects: a literature review. Aging Clin Exp Res. 2016:28(3):393-405.

41. Solloway MR, et al. An evidence map of the effect of tai chi on health outcomes. Syst Rev. 2016;5(1):126.

42. Wayne PM, et al. Effect of tai chi on cognitive performance in older adults: systematic review and meta-analysis. J Am Geriatr Soc. 2014;62(1):25-39.

43. Mortimer JA, et al. Changes in brain volume and cognition in a randomized trial of exercise and social interaction in a community-based sample of nondemented Chinese elders. J Alzheimers Dis. 2012;30(4):757-66.

44. Borde R, Hortobagyi T, Granacher U. Dose-response relationships of resistance training in healthy old adults: a systematic review and metaanalysis. Sports Med. 2015;45(12):1693-720.

45. Shigematsu $R$, et al. Dance-based aerobic exercise may improve indices of falling risk in older women. Age Ageing. 2002;31(4):261-6.

46. Dhami P, Moreno S, DeSouza JF. New framework for rehabilitation-fusion of cognitive and physical rehabilitation: the hope for dancing. Front Psychol. 2015:5:1478

47. Hamacher $D$, et al. Does dancing improve fall risk due to its demands on cognitive effort. In: Front. Hum. Neurosci. Conference Abstract: XI International Conference on Cognitive Neuroscience (ICON-XII); 2015. https://doi.org/10.3389/conf. fnhum.

48. Okubo Y, Schoene D, Lord SR. Step training improves reaction time, gait and balance and reduces falls in older people: a systematic review and meta-analysis. Br J Sports Med. 2017;51(7):586-593.

49. Schoene $D$, et al. Interactive cognitive-motor step training improves cognitive risk factors of falling in older adults - a randomized controlled trial. PLoS One. 2015;10(12):e014561.

50. Kattenstroth J-C, et al. Six months of dance intervention enhances postural, sensorimotor, and cognitive performance in elderly without affecting cardio-respiratory functions. Front Aging Neurosci. 2013;5:5.

51. Merom D, et al. Can social dancing prevent falls in older adults? A protocol of the dance, aging, cognition, economics (DAnCE) fall prevention randomised controlled trial. BMC Public Health. 2013;13(1):1.

52. Hamacher $D$, et al. The effect of a six-month dancing program on motorcognitive dual-task performance in older adults. J Aging Phys Act. 2015; 23(4):647-52.

53. Hwang PW-N, Braun KL. The effectiveness of dance interventions to improve older adults' health: a systematic literature review. Altern Ther Health Med. 2015;21(5):64. 
54. Gajewski PD, Falkenstein M. Physical activity and neurocognitive functioning in aging - a condensed updated review. Eur Rev Aging Phys Act. 2016;13:1

55. Brach JS, VanSwearingen JM. Interventions to improve walking in older adults. Curr Transl Geriatr Exper Gerontol Rep. 2013;2(4):230-8.

56. Tabei $\mathrm{K}-\mathrm{I}$, et al. Physical exercise with music reduces gray and white matte loss in the frontal cortex of elderly people: the Mihama-Kiho scan project. Front Aging Neurosci. 2017:9:174.

57. Healy AF, Kole JA, Bourne LE Jr. Training principles to advance expertise. Psychol Perspectives Expert. 2014:5:131.

58. Ammann $\mathrm{BC}$, et al. Application of principles of exercise training in sub-acute and chronic stroke survivors: a systematic review. BMC Neurol. 2014;14(1):1

59. Chodzko-Zajko WJ, et al. Exercise and physical activity for older adults. Med Sci Sports Exerc. 2009;41(7):1510-30.

60. Nelson ME, et al. Physical activity and public health in older adults: recommendation from the American College of Sports Medicine and the American Heart Association. Circulation. 2007;116(9):1094.

61. Paterson DH, Jones GR, Rice CL. Ageing and physical activity: evidence to develop exercise recommendations for older adults. Appl Physiol Nutr Metab. 2007;32(S2E):S69-S108.

62. Law LL, et al. Effects of combined cognitive and exercise interventions on cognition in older adults with and without cognitive impairment: a systematic review. Ageing Res Rev. 2014;15:61-75.

63. Brooke J. SUS-A quick and dirty usability scale. Usability Eval Indust. 1996; 189(194):4-7.

64. Vaziri DD, et al. Exploring user experience and technology acceptance for a fall prevention system: results from a randomized clinical trial and a living lab. Eur Rev Aging Phys Act. 2016;13(1):1.

65. Bangor A, Kortum P, Miller J. Determining what individual SUS scores mean: adding an adjective rating scale. J Usability Stud. 2009:4(3):114-23.

66. Chesham A, et al. What older people like to play: genre preferences and acceptance of casual games. JMIR Serious Games. 2017;5(2):e8.

67. IJsselsteijn, W., Y. De Kort, and K. Poels, The game experience questionnaire. Manuscript in preparation, 2008.

68. Lewis, C. and J. Rieman, Task-centered user interface design. A Practical Introduction, 1993.

69. De Bruin $\mathrm{E}$, et al. Feasibility of strength-balance training extended with computer game dancing in older people; does it affect dual task costs of walking. J Nov Physiother. 2011;1(104):2.

70. Nyman SR, Victor CR. Older people's participation in and engagement with falls prevention interventions in community settings: an augment to the Cochrane systematic review. Age Ageing. 2012;41(1):16-23.

71. de Bruin ED, et al. Quantification of everyday motor function in a geriatric population. J Rehabil Res Dev. 2007;44(3):417-28.

72. König $N$, et al. Is gait variability reliable? An assessment of spatio-tempora parameters of gait variability during continuous overground walking. Gait Posture. 2014:39(1):615-7.

73. Eggenberger $\mathrm{P}$, et al. Multicomponent physical exercise with simultaneous cognitive training to enhance dual-task walking of older adults: a secondary analysis of a 6-month randomized controlled trial with 1-year follow-up. Clin Interv Aging. 2015;10:1711-32.

74. Schättin A, et al. Adaptations of prefrontal brain activity, executive functions, and gait in healthy elderly following exergame and balance training: a randomized-controlled study. Front Aging Neurosci. 2016;8:278.

75. Jian Y, et al. Trajectory of the body COG and COP during initiation and termination of gait. Gait Posture. 1993;1(1):9-22.

76. McDowd JM. The effects of age and extended practice on divided attention performance. J Gerontol. 1986;41(6):764-9.

77. Guralnik JM, et al. Lower extremity function and subsequent disability: consistency across studies, predictive models, and value of gait speed alone compared with the short physical performance battery. J Gerontol A Biol Sci Med Sci. 2000;55(4):M221-31

78. Guralnik JM, et al. A short physical performance battery assessing lower extremity function: association with self-reported disability and prediction of mortality and nursing home admission. J Gerontol. 1994;49(2):M85-94.

79. Veronese $\mathrm{N}$, et al. Association between short physical performance battery and falls in older people: the progetto Veneto anziani study. Rejuvenation Res. 2014:17(3):276-84

80. Zimmermann P, Fimm B. A test battery for attentional performance. In: Applied neuropsychology of attention. Theory, diagnosis and rehabilitation; 2002. p. 110-51.
81. Jasper $\mathrm{H}$. Report of the committee on methods of clinical examination in electroencephalography. Electroencephalogr Clin Neurophysiol. 1958;10(2): 370-5.

82. Delorme A, Makeig S. EEGLAB: an open source toolbox for analysis of single-trial EEG dynamics including independent component analysis. J Neurosci Methods. 2004;134(1):9-21.

83. Grandy TH, et al. Peak individual alpha frequency qualifies as a stable neurophysiological trait marker in healthy younger and older adults. Psychophysiology. 2013;50(6):570-82.

84. Corcoran AW, et al. Toward a reliable, automated method of individual alpha frequency (IAF) quantification. Psychophysiology. 2018;55(7):e13064.

85. Cross, Z.R., et al., Alpha Oscillations Prior to Encoding Preferentially Modulate Memory Consolidation during Wake Relative to Sleep. bioRxiv, 2018

86. Cohen J. Statistical analysis for the behavioral sciences. Hillsdale: Lawrance Erlbaum; 1988.

87. Moher D, et al. CONSORT 2010 explanation and elaboration: updated guidelines for reporting parallel group randomised trials. Bmj. 2010;340:c869.

88. Brach $\mathrm{M}$, et al. Modern principles of training in exergames for sedentary seniors: requirements and approaches for sport and exercise sciences. Int J Comput Sci Sport. 2012;11(2012):86-99.

89. Bleakley CM, et al. Gaming for health a systematic review of the physical and cognitive effects of interactive computer games in older adults. J Appl Gerontol. 2015;34(3):NP166-89.

90. Silveira P, et al. Tablet-Based Strength-Balance Training to Motivate and Improve Adherence to Exercise in Independently Living Older People: A Phase II Preclinical Exploratory Trial. J Med Internet Res. 2013;15(8):e159.

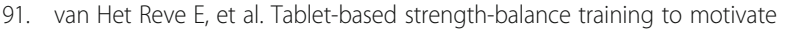
and improve adherence to exercise in independently living older people: part 2 of a phase II preclinical exploratory trial. J Med Internet Res. 2014; 16(6):e159.

92. Sjösten $N M$, et al. A multifactorial fall prevention programme in the community-dwelling aged: predictors of adherence. Eur J Public Health. 2007;17(5):464-70.

93. Valenzuela $\mathrm{T}$, et al. Adherence to technology-based exercise programs in older adults: a systematic review. J Geriatr Phys Ther. 2018:41(1):49-61.

94. Bamidis PD. Building neuroscientific evidence and creating best practices for Active and Healthy Aging through ubiquitous exergaming and Living Labs. In: 2015 37th Annual International Conference of the IEEE Engineering in Medicine and Biology Society (EMBC); 2015. IEEE.

95. Corbalan G, Kester L, van Merriënboer JJ. Dynamic task selection: effects of feedback and learner control on efficiency and motivation. Learn Instr. 2009; 19(6):455-65.

96. Gschwind YJ, et al. ICT-based system to predict and prevent falls (iStoppFalls): results from an international multicenter randomized controlled trial. Eur Rev Aging Phys Act. 2015;12:10.

97. Pichierri G, Murer K, de Bruin ED. A cognitive-motor intervention using a dance video game to enhance foot placement accuracy and gait under dual task conditions in older adults: a randomized controlled trial. BMC Geriatr. 2012;12:74

98. Beurskens $\mathrm{R}$, Bock O. Age-related deficits of dual-task walking: a review. Neural Plast. 2012;2012:131608.

99. Logghe $\mathrm{H}$, et al. The effects of tai chi on fall prevention, fear of falling and balance in older people: a meta-analysis. Prev Med. 2010;51(3):222-7.

100. Verhagen AP, et al. The efficacy of tai chi Chuan in older adults: a systematic review. Fam Pract. 2004;21(1):107-13.

101. Woo J, et al. A randomised controlled trial of tai chi and resistance exercise on bone health, muscle strength and balance in community-living elderly people. Age Ageing. 2007;36(3):262-8.

102. Morishita S, et al. Relationship between the rating of perceived exertion scale and the load intensity of resistance training. Strength Condition J. 2018:40(2):94-109.

103. Fragala MS, et al. Resistance Training for Older Adults: Position Statement From the National Strength and Conditioning Association. J Strength Cond Res. 2019;33(8):2019-52.

104. Eggenberger $P$, et al. Does multicomponent physical exercise with simultaneous cognitive training boost cognitive performance in older adults? A 6-month randomized controlled trial with a 1-year follow-up. Clin Interv Aging. 2015;10:1335-49.

105. Anderson-Hanley $C$, et al. Exergaming and older adult cognition a cluster randomized clinical trial. Am J Prev Med. 2012;42(2):109-19. 
106. Anguera JA, et al. Video game training enhances cognitive control in older adults. Nature. 2013;501(7465):97-101.

107. Klimesch W. EEG alpha and theta oscillations reflect cognitive and memory performance: a review and analysis. Brain Res Rev. 1999;29(2):169-95.

108. Vecchio F, et al. Resting state cortical EEG rhythms in Alzheimer's disease: toward EEG markers for clinical applications: a review. In: Supplements to Clinical neurophysiology: Elsevier; 2013. p. 223-36. https:/www. sciencedirect.com/bookseries/supplements-to-clinical-neurophysiology.

109. Luckhaus C, et al. Quantitative EEG in progressing vs stable mild cognitive impairment (MCI): results of a 1-year follow-up study. Int J Geriatr Psychiatry. 2008;23(11):1148-55.

110. Babiloni $C$, et al. Sources of cortical rhythms change as a function of cognitive impairment in pathological aging: a multicenter study. Clin Neurophysiol. 2006;117(2):252-68.

111. Thabane L, et al. A tutorial on pilot studies: the what, why and how. BMC Med Res Methodol. 2010;10:1

112. Arain $\mathrm{M}$, et al. What is a pilot or feasibility study? A review of current practice and editorial policy. BMC Med Res Methodol. 2010;10(1):67.

\section{Publisher's Note}

Springer Nature remains neutral with regard to jurisdictional claims in published maps and institutional affiliations.

Ready to submit your research? Choose BMC and benefit from:

- fast, convenient online submission

- thorough peer review by experienced researchers in your field

- rapid publication on acceptance

- support for research data, including large and complex data types

- gold Open Access which fosters wider collaboration and increased citations

- maximum visibility for your research: over $100 \mathrm{M}$ website views per year

At BMC, research is always in progress.

Learn more biomedcentral.com/submissions 